\title{
Estimativa numérica e desempenho aritmético
}

Numerical estimation and arithmetic performance

\author{
Mariana Lima Duro ${ }^{1}$ \\ Beatriz Vargas Dorneles ${ }^{2}$
}

\section{Resumo}

O presente estudo relacionou a precisão da estimativa numérica em dois testes de desempenho em estimativa numérica: o Teste de Estimativa Numérica de Quantidades (TENQ) e o Teste de Estimativa na Reta Numérica (TERN), com o desempenho em um teste padronizado de aritmética (TDE), em estudantes do $5^{\circ}$ ano escolar, de uma escola pública e outra particular de Porto Alegre/RS. O principal objetivo desse estudo foi verificar se o desempenho em diferentes tarefas de estimativa numérica está relacionado ao desempenho aritmético desses estudantes. Os dados indicam que o desempenho aritmético apresentou correlação de moderada a forte com o desempenho em estimativa, em ambas as tarefas, quando considerado o intervalo numérico de 0 a 100. Esse resultado indicou que conhecimentos aritméticos possam estar relacionados, de alguma forma, à habilidade em realizar estimativas.

Palavras chave: estimativa numérica de quantidades; estimativa na reta numérica; desempenho aritmético.

\section{Abstract}

The present study correlated the accuracy of the number estimation in two number estimation tests: the Numerosity Test (TENQ) and the Number Line Estimation Test (TERN), with the performance in a standardized arithmetic test (TDE), in students of the 5 th $-6^{\text {th }}$ grade of public and private school in Porto Alegre / RS. The main objective of this study was to verify if the performance in different tasks of numer estimation is related to the arithmetic performance of these students. The data indicate that the arithmetic performance presented a moderate to strong correlation with the performance in estimation in both tasks when considering the 0-100 numerical interval. This result indicated that arithmetical knowledge may be related, in some way, to the estimative ability.

Keywords: numerosity; number line estimation; arithmetic performance.

\footnotetext{
${ }^{1}$ Instituto Federal de Educação, Ciência e Tecnologia do Rio Grande do Sul | profmarianaduro@gmail.com

${ }^{2}$ Universidade Federal do Rio Grande do Sul | bvdornel@terra.com.br
} 


\section{Introdução}

Alguns conhecimentos matemáticos parecem estar muito naturalmente relacionados com a habilidade de realizar estimativas, tais como: a compreensão de valor posicional, o cálculo mental, a tolerância para o erro, a compreensão de propriedades aritméticas etc. Entretanto, muitas crianças consideram a estimativa como uma alternativa inferior ao cálculo exato, dificultando a aquisição de experiências e, consequentemente, da confiança em juízos de estimativa (AZEVEDO, 1996).

Nessa perspectiva, é importante entender a estimativa, em suas mais diferentes formas de realização, compreendendo qual tarefa é mais facilmente realizada pelos estudantes e qual o papel da estimativa no desempenho aritmético. Sendo assim, avaliar o desempenho da estimativa pode ser válido, tendo em vista que o seu desenvolvimento tem sido considerado um bom preditor de habilidades matemáticas simbólicas posteriores (PARK \& BRANNON, 2013). Então, comparar o desempenho em diferentes tarefas de estimativa com o desempenho matemático aritmético dos estudantes pode ser uma boa alternativa para compreender melhor essas relações.

Considera-se inovadora a proposta deste estudo, tendo em vista que a comparação do desempenho em estimativa numérica de crianças do $5^{\circ}$ e $6^{\circ}$ ano com o desempenho em aritmética dessas crianças, até onde se sabe, não foi investigada em outro estudo brasileiro. Aqui, buscou-se verificar se o desempenho nesses testes estaria relacionado ao desempenho aritmético dessas crianças em um teste padronizado de matemática (TDE). Teve-se por hipótese que ambos os testes devem refletir no desempenho em aritmética das crianças, tendo em vista as evidências de que estimativa e aritmética são habilidades intimamente relacionadas.

Por fim, a questão mais discutida na literatura considera a possibilidade de a estimativa refletir a forma como se representa mentalmente os números (reta numérica mental) ou como o desempenho em tarefas desse tipo pode ser afetado por um conhecimento limitado de número (EBERSBACH; LUWEL \& VERSCHAFFEL, 2015). Entretanto, a mais consistente conclusão é que as pessoas não são boas em realizar estimativas. Mesmo que já exista considerável tradição em estudos sobre este tema, no Brasil, até onde se sabe, a estimativa numérica não foi investigada e, nas pesquisas internacionais, ainda há pouco consenso sobre os resultados, embora muitos relatem a falta do seu ensino nas escolas. 0 cenário teórico descrito anteriormente demonstra a necessidade de mais estudos sobre o tema da estimativa numérica.

Esta pesquisa integra um projeto mais abrangente, intitulado "Diversidade na aprendizagem da matemática inicial: a compreensão da estimativa numérica" (Plataforma Brasil e Comitê de Ética em Pesquisa da Universidade Federal do Rio Grande do Sul, sob o número 31575913.6.0000.5347). O projeto tem como proposta o estudo da diversidade na aprendizagem da matemática, no que se refere à compreensão da estimativa numérica em diferentes grupos de alunos. 


\title{
Referencial Teórico
}

\author{
Representação Numérica Mental
}

Algumas evidências empíricas sugerem que alguns animais, humanos ou não, possuem uma representação interna de número tal como uma reta numérica mental (DEHAENE, 1997). A reta numérica é uma linha na qual os números estão dispostos em ordem crescente e separados em uma escala de magnitude pré-estabelecida. Para alguns autores, a estimativa na reta numérica pode oferecer conhecimentos importantes, não só sobre as representações mentais de número, mas também sobre o desenvolvimento numérico (MENZIES et al., 2013). A partir da tarefa em que as crianças são convidadas a posicionar números em uma reta numérica (BERTELETTI et al., 2010; BOOTH \& SIEGLER, 2006), os pesquisadores passaram a desenvolver modelos detalhados de como os números são representados mentalmente.

O Modelo Acumulador de Gibbon e Church (1981) propõe que a representação numérica mental é linear, sendo que as distâncias entre as magnitudes numéricas são iguais em escala. Ao contrário, o Modelo Logaritmo, proposto por Dehaene (1997), pressupõe que a representação numérica está em uma escala numérica mental comprimida para números maiores e com grande espaçamento entre números pequenos. Mais tarde, os pesquisadores passaram a sugerir a existência de uma dupla representação numérica, passando de logarítmica dominante no início do desenvolvimento e, com a idade e a experiência numérica, tornando-se cada vez mais linear (MENZIES et al., 2013), evidenciando que a distribuição das estimativas na reta numérica segue um padrão de mudança logarítmico-linear conforme as crianças ficam mais velhas (BOOTH \& SIEGLER, 2006, 2008; SIEGLER \& BOOTH, 2004; SIEGLER \& OPFER, 2003). As evidências empíricas para apoiar o modelo de mudança logarítmico-linear vêm em grande parte do Teste de Estimativa na Reta Numérica (TERN), proposto por Siegler e Booth (2004).

Ainda há relatos de que essa mudança de representação logarítmica-linear pode não estar apenas relacionada com a idade, mas também com o conhecimento numérico da criança. Essa constatação deu origem ao Modelo Duplo-linear. Este modelo sugere que as crianças podem apresentar uma representação mental linear quando o número a ser estimado encontra-se dentro de sua faixa de contagem e uma representação logarítmica para números além dessa faixa (BERTELETTI et al., 2010). Esta última hipótese já foi investigada por alguns pesquisadores (EBERSBACH et al., 2008; NUERK et al., 2004) que afirmaram que a chamada representação logarítmica observada em crianças menores seria, na verdade, duas funções lineares separadas, uma com um declive mais acentuado (para números menores) e outra com um leve declive (para números maiores). Entretanto, não há consenso sobre o ponto de ruptura entre os dois segmentos. Ebersbach e colaboradores (2008) sugerem que esse ponto é variável e caracterizado pelo final da série numérica à qual as crianças são familiarizadas. Em outras palavras, essa representação linear de números muda de acordo com a idade e a faixa numérica conhecida pelas crianças. Em contrapartida, Moeller e colaboradores (2009) consideram que esse ponto de interrupção é fixo e representa mudanças entre números de um e dois dígitos.

Um terceiro modelo foi proposto: o Juízo Proporcional (BARTH \& PALADINO, 2011). Neste modelo, a estimativa na reta numérica é vista como uma aplicação proporcional do comprimento total da reta. Inicialmente, estimam-se as magnitudes apenas confiando no ponto inicial e ponto final da reta numérica. Mais tarde, usam-se outros pontos de 
referência intermediários como auxílio para as estimativas. Assim, de acordo com o intervalo numérico, quanto mais pontos de referência forem disponibilizados, mais precisa é a estimativa, especialmente perto desses pontos.

Apesar do debate em curso (BARTH \& PALADINO, 2011; EBERSBACH et al., 2008; MOELLER et al., 2009) a resposta à pergunta sobre qual destes modelos de representação reflete melhor os padrões de evolução das estimativas, é inconclusiva. Para superar essa lacuna, Dackermann e colaboradores (2015) apresentaram uma proposta de integração desses modelos, cuja ideia é aqui compartilhada (DORNELES et al., 2017), sugerindo que a representação numérica pode refletir a compreensão das relações numéricas em suas diferentes etapas, considerando o intervalo numérico adotado, da idade do sujeito pesquisado e a sua familiaridade com o intervalo (SIEGLER \& BOOTH, 2004; BOOTH \& SIEGLER, 2006).

Embora haja diferenças importantes nas teorias de representação do número, são consensuais as conclusões obtidas até o momento de que a) as estimativas tornam-se mais precisas e lineares com a idade, b) o desempenho em estimativa está ligado de alguma forma ao desempenho matemático e c) tanto a estimativa quanto o desempenho de matemático estão relacionados com a capacidade de contar e à familiaridade com números.

\section{Estimativa na Reta Numérica e Desempenho Matemático}

A maior parte dos estudos envolvendo estimativa numérica utiliza a reta numérica com instrumento para comparar magnitudes. A tarefa da reta numérica envolve a compreensão do sistema numérico, as representações mentais de quantidade numérica e as estratégias de mapeamento de informações numéricas espaciais (SULLIVAN et al., 2011). O grupo de pesquisadores que defende que o desempenho na tarefa da reta numérica reflete a representação numérica mental (DEHAENE et al., 2008; SIEGLER \& OPFER, 2003) e que essa representação pode fornecer a estrutura conceitual para o desenvolvimento de conhecimentos numéricos mais complexos (BOOTH \& SIEGLER, 2008) foi contestado (WHITE \& SZUCS, 2012).

Sobre o fato de as estimativas tornarem-se mais lineares e, por isso, mais precisas com a idade, Siegler e Opfer (2003) trouxeram um estudo bastante conclusivo que fornece evidências de que alunos da $2^{a}$ série apresentam padrão logarítmico de representação numérica, na $4^{a}$ série os alunos apresentam tanto padrão logarítmico como linear e, a partir da $6^{a}$ série, passam a apresentar unicamente um modelo linear de representação, especialmente na reta 0-1000. Complementando esses achados, Siegler e Booth (2004) verificaram que os padrões de estimativas progridem de uma forma logarítmica em préescolares a uma mistura de logarítmica e linear na $1^{a}$ série a, finalmente, um padrão principalmente linear na $2^{a}$ série, em se tratando de uma escala na reta 0-100. Replicando os estudos de Siegler e Opfer (2003), Opfer e Siegler (2007) chegaram às mesmas conclusões, aplicando uma gama mais ampla de números a serem posicionados na reta numérica.

Em estudo intercultural, Xu e colaboradores (2013) também concluíram que a representação linear de números aumenta com a idade, mas que crianças chinesas já possuem representações lineares desde muito cedo, contradizendo a hipótese levantada por Siegler e Opfer (2003), de que a estimativa numérica é uma medida pura, e concluindo que a cultura possibilita que as crianças sejam mais hábeis em realizar estimativas. Na pesquisa de Siegler e Mu (2008), crianças chinesas apresentam escalas numéricas mais lineares antes do que estadunidenses, ao contrário dos achados de Muldoon e 
colaboradores (2011), cujos dados evidenciaram que crianças chinesas não apresentam escalas numéricas mais lineares (e, portanto, mais precisas) antes que crianças escocesas, com habilidade matemática equivalente.

Uma conclusão consistente sobre a relação entre linearidade das estimativas e precisão foi encontrada por Berteletti e colaboradores (2010), que confirmaram que as estimativas das crianças passaram de logarítmicas a lineares, sendo que elas se tornaram menos precisas, mas cada vez mais logarítmicas para intervalos numéricos em que não estivessem familiarizadas. E, em contraste com as alegações anteriores, de que a linearidade da representação numérica é uma contribuição única para o desenvolvimento matemático das crianças, os dados de Menzies e colaboradores (2013) sugeriram que essa variável não é significativamente superior sobre habilidades numéricas básicas.

Mais especificamente, a correlação da estimativa na reta numérica com o desempenho em aritmética foi destacada por diversos pesquisadores (SIEGLER \& BOOTH, 2004; BOOTH \& SIEGLER, 2008; SIEGLER \& MU, 2008; SCHNEIDER; GRABNER \& PAETSCH, 2009; MULDOON et al., 2011; BOOTH \& SIEGLER, 2006; LEFEVRE et al., 2013). Siegler e Booth (2004) encontraram uma correlação entre tarefas de estimativa e um teste padronizado de desempenho matemático, mesmo após o controle da inteligência e da idade. Replicando esses achados, Schneider, Grabner e Paetsch (2009) concluíram que o conhecimento conceitual, a inteligência numérica e a estimativa na reta numérica foram bons preditores do desempenho matemático. Booth e Siegler (2006) concluíram que o desempenho em estimativa foi capaz de predizer o desempenho em aritmética e, mais tarde (BOOTH \& SIEGLER, 2008), concluíram que a linearidade das representações da magnitude numérica ainda previa a aprendizagem aritmética. Corroborando estes achados, Menzies e colaboradores (2013) encontraram mais evidências de que estimativa na reta numérica, habilidades matemáticas e procedimentos de contagem estão correlacionados. Berteletti e colaboradores (2010) destacaram a correlação entre a precisão da estimativa e o conhecimento de algarismos arábicos e ordem numérica.

Em suas discussões, Siegler e Booth (2004) sugerem que as representações de magnitudes numéricas das crianças incluem um componente espacial forte, de tal forma que números maiores são posicionados em condições espacialmente maiores. Da mesma maneira, Lefevre e colaboradores (2013) encontraram que a capacidade espacial foi correlacionada com todas as medidas em matemática em um estudo com estudantes da $3^{a}$ e $4^{a}$ séries. Esta pode ser uma razão para que crianças, a partir dos 5 anos, realizem a tarefa de estimativa na reta numérica de maneira razoável, depois de 30 segundos.

Contradizendo a maior parte dos estudos em estimativa na reta numérica, Lefevre e colaboradores (2013) não encontraram evidências de que o desempenho na reta numérica é preditivo para cálculo mais do que o cálculo é preditivo do desempenho em estimativa na reta numérica. Ou seja, há divergências sobre esta relação causal e os estudos da área ainda são inconclusivos.

\section{Estimativa Numérica de Quantidades e Desempenho Matemático}

Algumas pesquisas têm demonstrado que o desempenho em estimativa numérica se correlaciona com testes aritméticos padronizados (BOOTH \& SIEGLER, 2006; SIEGLER \& BOOTH, 2004), tarefas de adição simples (SIEGLER \& MU, 2008) e também com processos numéricos específicos, tais como aritmética e comparação de magnitude (SIEGLER \& BOOTH, 2005; LASKI \& SIEGLER, 2007). Ressalta-se que Muldoon e colaboradores (2011) 
encontraram resultados contrários em um estudo sobre a capacidade matemática de crianças ocidentais e orientais em que a linearidade e exatidão mostravam-se independentes da habilidade matemática.

Desempenho aritmético é uma construção complexa, não só composta por cálculos como adição e subtração, mas também pela capacidade de operar com números de uma forma flexível (OBERSTEINER et al., 2014). Sendo assim, conhecimento sobre números pode restringir a quantidade de possíveis respostas a problemas aritméticos, excluindo respostas improváveis e qualificando a gama de outras mais prováveis. Em 1982, Levine (1982) constatou que os alunos com boa capacidade aritmética usam estimativa com mais frequência do que aqueles com pior capacidade aritmética. Os achados atuais indicam que as representações de magnitudes numéricas não são apenas positivamente relacionadas com uma variedade de tipos de conhecimento numérico, mas também preditivo de sucesso na aquisição de novos dados numéricos, em particular, respostas para problemas aritméticos (BOOTH \& SIEGLER, 2008).

Quanto à relação entre a estimativa numérica de quantidades e o desempenho em matemática, Booth e Siegler (2006) encontraram correlação positiva para alunos da $3^{a}$ e $4^{a}$ séries, embora não para os alunos da $1^{a}$ e $2^{a}$ séries. Gilmore, Mccarthy e Spelke (2010) verificaram que a precisão das crianças na tarefa não-simbólica se correlacionou positivamente com a sua habilidade matemática. Da mesma maneira, Obersteiner e colaboradores (2014) mostraram que o desempenho na tarefa de estimativa de quantidades foi correlacionado com o desempenho em um teste de aritmética, mesmo quando outras variáveis cognitivas foram controladas estatisticamente. Ao contrário, Park e Brannon (2013) relatam que uma melhoria substancial na tarefa de aritmética aproximada resultou em uma melhoria também substancial na capacidade matemática simbólica, sugerindo uma mudança de causalidade entre as habilidades de estimativa e as habilidades aritméticas.

Lipton e Spelke (2005) trouxeram a questão da familiaridade das crianças com o intervalo numérico das quantidades apresentadas como fator essencial para a discriminação das quantidades. Nessa perspectiva, Booth e Siegler (2006) sugeriram que representações não-lineares podem ser uma fonte de dificuldade na aprendizagem de matemática, pois, em estudo desenvolvido pelos autores, a qualidade das estimativas em uma tarefa na reta numérica correlacionou-se com a capacidade do desempenho em problemas de adição simples. Este tipo de correlação eleva a preocupação sobre o desenvolvimento de representações lineares que podem influenciar na aprendizagem matemática.

Em termos de padrão mental de representação, Thompson e Siegler (2010) concluíram que as crianças, cuja estimativa tinha padrão linear, utilizavam mais a representação logarítmica para números além do intervalo numérico em que estivessem familiarizados. Um fator importante para esse estudo são as diferenças relacionadas à idade, tendo em vista que se considera que existam diferentes níveis de desenvolvimento da estimativa desde a infância até a estimativa adulta, mais precisa. Booth e Siegler (2006) afirmam que a estimativa de quantidades muda com a idade (paralelamente ao desenvolvimento de estimativas na reta numérica).

Da mesma maneira, Gandini, Lemaire e Dufau (2008) buscaram compreender os processos de estimativa e suas mudanças com a idade e seus dados forneceram evidências de que os jovens são quase sempre mais rápidos e mais precisos do que os idosos. Ao contrário dessas pesquisas, Huntley-Fenner (2001) encontrou estimativas semelhantes para crianças e adultos, porém o desvio padrão das estimativas parecia diminuir com a idade. Corroborando estes achados, Lemaire e Lecacheur (2007) concluíram que adultos e idosos 
apresentam desempenho comparável e que não há diferenças relacionadas à idade para estimativa de quantidades, a não ser que os participantes mais velhos levam mais tempo do que os jovens adultos para fornecer suas estimativas. Ou seja, a representação simbólica e não-simbólica de números pode coexistir a partir do início do desenvolvimento, mas têm diferentes cursos no decorrer do desenvolvimento.

\section{Método}

Comparou-se o desempenho em estimativa numérica com o desempenho aritmético em um grupo de 284 estudantes do $5^{\circ}(\mathrm{N}=138)$ e $6^{\circ}(\mathrm{N}=146)$ ano escolar, que formavam a população de duas escolas: uma escola pública municipal de ensino fundamental ( $N=89$ $5^{\circ}$ ano e $\mathrm{N}=77-6^{\circ}$ ano e uma escola privada ( $\mathrm{N}=49-5^{\circ}$ ano e $\mathrm{N}=69-6^{\circ}$ ano), ambas na cidade de Porto Alegre/RS.

\section{Instrumentos de Coleta de Dados}

Para coleta de dados, foram utilizados dois testes de avaliação de desempenho em estimativa numérica: a) o Teste de Estimativa Numérica de Quantidades (TENQ), adaptado de Luwel e Verschaffel (2003), que consistia em estimar pontos distribuídos em matrizes 10x1, 10x2 e 10x10 e b) o Teste de Estimativa na Reta Numérica (TERN), adaptado de Siegler e Booth (2004), que consistia e em estimar o posicionamento de números em reta numéricas de intervalos 0-10, 0-20 e 01-100 e um teste de avaliação de desempenho aritmético (TDE - Subteste de aritmética), de Stain (1994).

O Teste de Desempenho Escolar - Subteste de Aritmética (TDE): Para avaliação de desempenho em aritmética, foi utilizado o Teste de Desempenho Escolar (TDE) - Subteste de Aritmética, desenvolvido por Stein (1994), com o objetivo de avaliar habilidades de cálculo aritmético. A escolha deste subteste considerou, principalmente, a possibilidade de aplicação coletiva em estudantes do $3^{\circ}$ ao $7^{\circ}$ ano do Ensino Fundamental, a obtenção dos padrões estatísticos estabelecidos a partir de seus resultados e por sua padronização ter sido realizada com populações socioeconômicas e culturais semelhantes as dos sujeitos do estudo.

Este subteste é composto de duas partes: uma parte oral e outra parte de escrita de cálculos. A parte oral contém três problemas que envolvem comparação de quantidades e operações de adição e subtração simples. A outra parte envolve 35 questões de operações de adição, subtração, multiplicação, divisão e potenciação com números naturais, decimais, fracionários e inteiros. O teste inclui uma tabela de classificação de desempenho (inferior, médio ou superior) para cada ano de escolaridade. Para obter o resultado do teste, basta contabilizar a quantidade de acertos. Por tratar-se de um teste comercializado, de autoria de terceiros, não é possível disponibilizá-lo nos anexos do estudo.

\section{Análise dos Dados}

Para análise dos dados, as variáveis envolvidas nos testes de estimativa foram comparadas à variável independente precisão relativa (PR) e desempenho em aritmética (DA), obtido pelo somatório de pontos do TDE - Subteste de Aritmética (TDE). Para o cálculo da PR, utilizou-se a fórmula adaptada de Siegler e Booth (2004), sendo assim, 
considerou-se como "Valor Real" o valor real a ser estimado e "Estimativa" a resposta dada pela criança, então: $P R=\frac{\mid \text { VALOR REAL-ESTIMATIVA } \mid}{\text { ESCALA (10,20 ou 100) }}$.

Para realizar a avaliação de desempenho em aritmética, inicialmente foi calculada a quantidade de acertos de questões do TDE, para verificar quais as questões de aritmética eram mais frequentemente acertadas pelas crianças e quais apresentavam maior percentual de erro. Essa resposta é importante para, no caso de haver correlação entre o desempenho no teste de aritmética e o desempenho nas tarefas de estimativa, possa ser possível identificar quais tarefas de aritmética podem estar relacionadas às habilidades de estimativa numérica.

\section{Resultados e discussão}

A menos que indicado de outra forma, um nível de confiança alfa de 0,05 foi utilizado para todos os testes estatísticos. Os p-valores exatos foram relatados, mas valores muito pequenos foram arredondados para $p<0,001$. A média geral de acertos das 35 questões do TDE dentre os estudantes de $5^{\circ}$ e $6^{\circ}$ ano de ambas as escolas foi de 18,11 (51,43\%). Constatou-se que as questões mais acertadas, com mais de $90 \%$ das respostas corretas, foram as questões de soma e subtração com um algarismo, apresentadas de duas maneiras: por cálculos escritos horizontalmente e por algoritmo, multiplicação com um algarismo e soma com duas parcelas com números de dois algarismos em cada uma. Acima da média ainda tiveram as questões de subtração com "empréstimo", multiplicação e soma de números com mais de um algarismo e divisão exata com dividendo de dois algarismos e divisor com um número de um algarismo. As questões com maior frequência de erros envolviam combinação de soma com subtração, números decimais, frações, potenciação e operações com números negativos. As que obtiveram menos de $10 \%$ de acertos envolviam divisão com resposta decimal, potência de expoente dois, multiplicação de números negativos, soma de frações de numeradores distintos, soma de potências e divisão de frações.

Para determinar a relação entre os dois testes foi realizada uma correlação de Spearman. A precisão foi correlacionada com o desempenho obtido pelos estudantes no TDE. Correlações entre 0,6 a 0,9 são classificadas como forte e entre 0,9 e 1,0 são classificadas como muito forte. Esses dados aparecem descritos na Tabela 1.

Analisando as correlações encontradas, verificou-se que elas ocorrem unicamente de maneira fraca ou moderada, ou seja, não há forte correlação entre os instrumentos. Separadamente por escala e por ano escolar, destacou-se, na escala 10, na escola particular, correlação moderada no $6^{\circ}$ ano entre TDE e TERN. Já na escola particular, ambas as tarefas de TENQ foram moderadamente correlacionadas com o TDE, no $5^{\circ}$ ano. Entretanto, as escalas 10 e 20 pouco mostram sobre uma possível correlação entre os instrumentos, demonstrando, talvez, serem escalas que permitam estimativas mais intuitivas em todas as tarefas, trazendo poucas informações sobre um possível preditor de habilidades estimativas ou aritmética dos estudantes.

Quando comparada às demais escalas, a escala 100 é a que pode trazer maior quantidade de correlações entre os instrumentos e, em sua maioria, correlações concordantes em ambas as escolas. Em síntese, tanto na escola pública quanto na escola particular obteve-se correlação moderada no $5^{\circ}$ ano entre TENQ-A e TERN com TDE. Na 
escola particular ainda foi encontrada correlação entre TENQ-E e TDE, no $5^{\circ}$ ano. Resumindo, em E100 obteve-se correlações, porém distintas para $5^{\circ}$ e $6^{\circ}$ ano. Estes achados revelam que padrões de diferenças individuais e de desenvolvimento também estão presentes em tarefas numéricas de estimativa.

Tabela 1 - Correlação dos instrumentos TENQ-A, TENQ-E, TERN por ano e escala

\begin{tabular}{|c|c|c|c|c|}
\hline \multirow{3}{*}{ Escala } & \multirow{3}{*}{ Ano } & \multirow{3}{*}{ Instrumento } & $\mathrm{Pu}$ & $\mathrm{Pa}$ \\
\hline & & & TDE & TDE \\
\hline & & & $r(p)$ & $r(p)$ \\
\hline \multirow{6}{*}{10} & \multirow{3}{*}{$5^{\circ}$} & TENQ-A & $\begin{array}{l}-0,089 \\
(0,556)\end{array}$ & $\begin{array}{c}-0,216 \\
(0,058)\end{array}$ \\
\hline & & TENQ-E & $\begin{array}{l}-0,254 \\
(0,088)\end{array}$ & $\begin{array}{l}-0,028 \\
(0,810)\end{array}$ \\
\hline & & TERN & $\begin{array}{l}-0,084 \\
(0,577)\end{array}$ & $\begin{array}{l}-0,231 \\
(0,041)\end{array}$ \\
\hline & \multirow{3}{*}{$6^{\circ}$} & TENQ-A & $\begin{array}{l}-0,014 \\
(0,907)\end{array}$ & $\begin{array}{c}0,049 \\
(0,697)\end{array}$ \\
\hline & & TENQ-E & $\begin{array}{l}-0,159 \\
(0,196)\end{array}$ & $\begin{array}{c}0,060 \\
(0,633)\end{array}$ \\
\hline & & TERN & $\begin{array}{r}-0,139 \\
(0,259) \\
\end{array}$ & $\begin{array}{l}-0,333 \\
(0,006) \\
\end{array}$ \\
\hline \multirow{6}{*}{20} & \multirow{3}{*}{$5^{\circ}$} & TENQ-A & $\begin{array}{l}-0,238 \\
(0,111)\end{array}$ & $\begin{array}{l}-0,309 \\
(0,006)\end{array}$ \\
\hline & & TENQ-E & $\begin{array}{c}0,021 \\
(0,892)\end{array}$ & $\begin{array}{l}-0,314 \\
(0,005)\end{array}$ \\
\hline & & TERN & $\begin{array}{l}-0,198 \\
(0,188)\end{array}$ & $\begin{array}{l}-0,139 \\
(0,226)\end{array}$ \\
\hline & \multirow{3}{*}{$6^{\circ}$} & TENQ-A & $\begin{array}{l}-0,091 \\
(0,462)\end{array}$ & $\begin{array}{l}-0,153 \\
(0,220)\end{array}$ \\
\hline & & TENQ-E & $\begin{array}{l}-0,065 \\
(0,599)\end{array}$ & $\begin{array}{l}-0,025 \\
(0,843)\end{array}$ \\
\hline & & TERN & $\begin{array}{l}-0,250 \\
(0,040) \\
\end{array}$ & $\begin{array}{l}-0,033 \\
(0,790) \\
\end{array}$ \\
\hline \multirow{6}{*}{100} & \multirow{3}{*}{$5^{\circ}$} & TENQ-A & $\begin{array}{l}-0,304 \\
(0,040)\end{array}$ & $\begin{array}{l}-0,299 \\
(0,008)\end{array}$ \\
\hline & & TENQ-E & $\begin{array}{l}-0,155 \\
(0,304)\end{array}$ & $\begin{array}{l}-0,301 \\
(0,007)\end{array}$ \\
\hline & & TERN & $-0,465(0,001)$ & $\begin{array}{c}-0,462 \\
(<0,001)\end{array}$ \\
\hline & \multirow{3}{*}{$6^{\circ}$} & TENQ-A & $\begin{array}{l}-0,152 \\
(0,215)\end{array}$ & $\begin{array}{l}-0,175 \\
(0,160)\end{array}$ \\
\hline & & TENQ-E & $\begin{array}{l}-0,290 \\
(0,016)\end{array}$ & $\begin{array}{l}-0,079 \\
(0,529)\end{array}$ \\
\hline & & TERN & $\begin{array}{l}-0,270 \\
(0,026)\end{array}$ & $\begin{array}{l}-0,087 \\
(0,489)\end{array}$ \\
\hline
\end{tabular}

Este estudo populacional quantitativo e comparativo, realizado com crianças do $5^{\circ}$ e $6^{\circ}$ ano em duas escolas, uma pública e uma privada, teve como objetivo analisar a relação 
entre o desempenho em dois testes de estimativa numérica ao desempenho em aritmética, a partir de um teste de aritmética padronizado.

Relacionando as duas tarefas de estimativa (TENQ e TERN) com o TDE, para a escala 100, o desempenho em TENQ sempre esteve correlacionado com o desempenho em TERN, bem como, o desempenho de ambos também apresentaram correlação forte ou moderada com o TDE. Ou seja, nesta escala, as crianças que são habilidosas em um tipo de estimativa tendem a ser mais hábeis em outra tarefa de estimativa numérica, mostrando avanços paralelos para anos escolares semelhantes. Da mesma maneira, o desempenho em estimativa esteve moderadamente correlacionado ao desempenho aritmético.

As diferenças encontradas nos estudos sobre correlação entre habilidades de estimativa e aritmética (BOOTH \& SIEGLER, 2006; SIEGLER \& MU, 2008; MULDOON et al., 2011) comprovam ligações entre as habilidades espaciais e conhecimentos do sistema numérico (pelo menos para o intervalo especificado), requerendo conhecimentos e processos de ambos os domínios. Por isso, parece bastante provável que, mesmo que ambos os testes meçam habilidades de estimativa, as moderadas correlações entre as os testes podem indicar que suas tarefas estejam relacionadas a algumas funções cognitivas subjacentes diferentes.

\section{Considerações Finais}

Este estudo teve como objetivo comparar o desempenho de estudantes do $5^{\circ}$ e $6^{\circ} \mathrm{em}$ duas tarefas de estimativa com o desempenho em aritmética. Entretanto, ao contrário do que era esperado, os argumentos estatísticos que indicaram que os testes de estimativa estão correlacionados com o desempenho aritmético dos estudantes não foram consistentes, apresentando correlação moderada e fraca apenas no intervalo numérico de 0 a 100. Com esse resultado, discutir possibilidades para o aprimoramento de estratégias de estimativas pode ser interessante para o desenvolvimento aritmético para grandes números e quantidades.

Compararam-se os resultados obtidos nos dois testes de estimativa com o desempenho em aritmética, a partir do TDE. Tinha-se como hipótese que ambos os testes poderiam refletir o desempenho em aritmética das crianças, tendo em vista as evidências de que estimativa e aritmética são habilidades que têm se mostrado intimamente relacionadas. Nos dados da pesquisa, não foi encontrado respaldo para a afirmação de que o TERN ou o TENQ sejam correlacionados com o conhecimento em aritmética ou o contrário, em conformidade com outros estudos ainda inconclusivos, mas correlações moderadas a fortes foram estabelecidas quando considerado o intervalo numérico de 0 a 100.

Esta última afirmação abre a discussão para pesquisas futuras, sugerindo ampliação de escala na comparação entre tarefas. Entretanto, ressalta-se a ideia de que estratégias de estimativa numérica nos seus mais diversos tipos de tarefas devem ser discutidas em sala de aula. Mesmo que estudos futuros cheguem a conclusões consistentes de que não há uma relação de causalidade entre estimativa e contagem exata, somente a frequência do uso cotidiano desta habilidade já justificaria essas discussões.

Para pesquisas futuras, sugere-se, ainda, verificar e analisar os processos cognitivos envolvidos em ambas as tarefas, para compreender se seria possível ou não estabelecer uma relação de causalidade entre estimativa e aritmética. Sabe-se que variações possíveis das tarefas, como quando se aplicam quantidades numéricas maiores, estimar números já 
posicionados ou quantidades sugeridas a grupos de itens, também poderiam mostrar resultados diferentes.

Não foi controlado o conhecimento do sistema numérico dos sujeitos, embora tenhase fortes indícios de que todas as crianças estivessem familiarizadas com os três intervalos numéricos apresentados. Não foi discutido se a tarefa de estimativa na reta numérica reflete ou não uma reta numérica mental interna, uma vez que este não era o objetivo do estudo. Da mesma forma, não se discutiu sobre os modelos de representação interna nas conclusões sobre as análises. Provas relacionadas à velocidade com que as crianças realizaram as estimativas não foram testadas neste estudo, considerando que o tempo de execução de cada teste foi o mesmo para todos os participantes.

\section{Referências}

AZEVEDO, Maria Manuela Duarte de Oliveria. A aprendizagem da Estimação Matemática: um estudo no 20 ciclo. Dissertação de Mestrado. Lisboa: Universidade Nova de Lisboa, 1996.

BARTH, Hilary; PALADINO, Annie. The Development of Numerical Estimation: Evidence Against a Representational Shift. Developmental Science, 14, p. 125- 135, 2011.

BERTELETTI, Ilaria. et al. Numerical Estimation in Preschoolers. Developmental Psychology, 46 (2), p.545-551, 2010.

BOOTH, Julie; SIEGLER, Robert. Developmental and Individual Differences in Pure Numerical Estimation. Developmental Psychology, 41, p. 189-201, 2006.

BOOTH, Julie; SIEGLER, Robert. Numerical Magnitude Representations Influence Arithmetic Learning. Child Development, 79 (4), p.1016 - 1031, 2008.

DACKERMANN, Tanja. et al. An Integration of Competing Accounts on Children's Number Line Estimation. Frontiers in Psychology, v. 6, n. 884, 2015.

DEHAENE, Stanisla. et al. Log or Linear? Distinct Intuitions of the Number Scale in Western and Amazonian Indigene Cultures. Stanislas Science, 320, p. 1217, 2008.

DEHAENE, Stanislas. The Number Sense: How the mind creates mathematics. New York: Oxford University Press, 1997.

DORNELES, Beatriz Vargas. et al. Number Estimation in Children: an assessment study with number line estimation and numerosity tasks. In Congress of the European Society for Research in Mathematics Education (CERME), 10., 2017, Dublin City University, 2017.

EBERSBACH, Mirjam. et al. The Relationship Between the Shape of the Mental Number Line and Familiarity with Numbers in 5- to 9-year old children: evidence for a segmented linear model. Journal of Experimental Child Psychology, 99, p.1-17, 2008.

EBERSBACH, Mirjam; LUWEL, Koen; VERSCHAFFEL, Lieven. The Relationship Between Children's Familiarity with Numbers and Their Performance in Bounded and Unbounded Number Line Estimations. Mathematical Thinking and Learning, 17:2-3, p.136-154, 2015.

GANDINI, Delphine; LEMAIRE, Patrick; DUFAU, Stéphane. Older and Younger Adults' Strategies in Approximate Quantification. Acta Psychologica, 129, p.175-189, 2008. 
GIBBON, John; CHURCH, Russell. M. Time Left: Linear Versus Logarithmic Subjective Time. Journal of Experimental Psychology: Animal Behavior Processes, 7(2), p.87-108, 1981.

GILMORE, Camilla; MCCARTHY, Shannon; SPELKE, Elizabeth. Non-symbolic Arithmetic Abilities and Achievement in the First Year of Formal Schooling in Mathematics. Cognition, 115(3), p. 394-406, 2010.

HUNTLEY-FENNER, Gavin. Children's Understanding of Number is Similar to Adults' and Rats': numerical estimation by 5 7-year-olds. Cognition, 78, p. B27-B40, 2001.

LASKI, Elizabeth.; SIEGLER, Robert. Is 27 a Big Number? Correlational and Causal Connections Among Numerical Categorization, Number Line Estimation, and Numerical Magnitude Comparison. Child Development, 78 (6), p. 1723 - 1743, 2007.

LEFEVRE, Jo-Anne. et al. Charting the Role of the Number Line in Mathematical Development. Front. Psychol, 4, 2013.

LEMAIRE, Patrick; LECACHEUR, Mireille. Aging and Numerosity Estimation. Journal of Gerontology: Psychological Sciences, 62B (6), p. 305-312, 2007.

LEVINE, Deborah. Strategy Use and Estimation Ability of College Students. Journal for Research in Mathematics Education, 13, p. 350-359, 1982.

LIPTON, Jeniffer; SPELKE, Elizabeth. Preschool Children's Mapping of Number Words to Nonsymbolic Numerosities. Child Development, 76:5, p. 978 - 988, 2005.

LUWEL, Koen; VERSCHAFFEL, Lieven. Adapting Strategy Choices to Situational Factors: the effect of time pressure on children's numerosity judgment strategies. Psychologica Belgica, 2003.

MENZIES, Victoria. et al. A Longitudinal Analysis of Estimation, Counting Skills, and Mathematical Ability Across the First School Year. Developmental Psychology, 49 (2), p. 250257, 2013PlosOne, 6 (9), 2013.

MOELLER, Korbinian. et al. Children's Early Mental Number Line: Logarithmic or decomposed linear? Journal of Experimental Child Psychology, 103 (4), p. 503-515, 2009.

MULDOON, Kevin. et al. Cross-Cultural Comparisons of 5-Year-Olds' Estimating and Mathematical Ability. Journal of Cross-Cultural Psychology, 42(4), p. 669-681, 2011.

NUERK, Hans-Christoph. et al. On the Development of the Mental Number Line: more, less or never holistic with increasing age? Developmental Psychology, 40(6), p. 1199-1211, 2004.

OBERSTEINER, Andreas. et al. Do First Graders Make Efficient Use of External Number Representations? The Case of the Twenty-Frame. Cognition and Instruction, 32(4), p. 353373, 2014.

OPFER, John; SIEGLER, Robert. Representational Change and Children's Numerical Estimation. Cognitive Psychology, 55, p. 169-195, 2007.

PARK, Joonkoo; BRANNON, Elizabeth. Training the Approximate Number System Improves Math Proficiency. Psychol Sci, 24(10), p. 2013-9, 2013.

SCHNEIDER, Michael; GRABNER, Roland; PAETSCH, Jennifer. Mental Number Line, Number Line Estimation, and Mathematical Achievement: Their interrelations in grades 5 and 6. Journal of Educational Psychology, 101, p. 359-372, 2009. 
SIEGLER, Robert.; MU, Yan. Chinese Children Excel on Novel Mathematics Problems Even Before Elementary School. Psychological Science, 19, p. 759-763, 2008.

SIEGLER, Robert; BOOTH, Julie. Development of Numerical Estimation in Young Children. Child Development, 75, p. 428-444, 2004.

SIEGLER, Robert; BOOTH, Julie. Development of Numerical Estimation: A Review. In CAMPBELL, J. I. D. Handbook of Mathematical Cognition. Psychology Press: New York. Cap. 2, p. 197-212, 2005.

SIEGLER, Robert; OPFER, John. E. The Development of Numerical Estimation: Evidence for multiple representations of numerical quantity. Psychological Science, 14, p. 237-243, 2003.

STEIN, Lilian. TDE: Teste de Desempenho Escolar: manual para a aplicação e interpretação. São Paulo: Casa do Psicólogo, 1994.

SULLIVAN, Jessica. et al. Adults' Number-line Estimation Strategies: Evidence from eye movements. Psychon Bull Rev, 18, p. 557-563, 2011.

THOMPSON, Clarissa; SIEGLER, Robert. Linear Numerical-Magnitude Representations Aid Children's Memory for Numbers. Psychological Science, 21(9), p. 1274-1281, 2010.

WHITE, Sonia; SZÜC, Dènes. Representational Change and Strategy use in Children's Number Line Estimation During the First Years of Primary School. Behavioral and Brain Functions, 8:1, 2012

$\mathrm{XU}$, Xiaohui. et al. Development of Numerical Estimation in Chinese Preschool Children. Journal of Experimental Child Psychology, 116, p. 351-366, 2013. 